\title{
Experimental Research about Shock Wave in a 1+1/2 Counter-Rotating Turbine
}

\author{
Chao $\mathrm{Li}^{1}$, Huo-Xing $\mathrm{Liu}^{1,2}$ and Zhi-Hong Zhou ${ }^{1}$ \\ ${ }^{1}$ School of Energy and Power Engineering, Beihang University, Beijing, China. \\ ${ }^{2}$ Defense aerothermodynamic laboratory \& School of Energy and Power Engineering, Beihang University, Beijing, China.
}

\begin{abstract}
To investigate the internal distribution regularities of shock wave structure in $1+1 / 2$ counter-rotating turbine, numerical simulation and experimental research about the shock wave structure were conducted by using the schlieren apparatus under different working conditions.From the point of the unsteady results, the unsteady effect has few influence on the flow field of high pressure guide vane, but the wake of the high pressure guide leaves periodically sweeps through the front edge of the high pressure blade and there presents strong unsteady effect on flow field of high pressure rotor. Because of periodic influence of external wake and shock wave, the unsteadiness of flow in low pressure rotor is still strong but not that drastic compared to the high pressure rotor. $50 \%$ height section of the blade of the three types of blades are extracted respectively to make plane cascades which are conducted blowing experiments in supersonic wind tunnel. The final photograph were analyzed by comparing with the CFD results. Results show that with the increase of expansion ratio, the wave structures in blade channel move toward the exit and the caudal interference between the outer tail wave and is strengthened gradually.
\end{abstract}

\section{Introduction}

In $1+1 / 2$ counter-rotating turbine, the high-pressure turbine has one stage, which contains a complete guide vane and a rotor blade. The low-pressure turbine also has one stage but only the rotating blade without guide vanes. The corresponding velocity triangle of the $1+1 / 2$ counterrotating turbine and the conventional $1+1$ counterrotating turbine are shown in Fig. 1.
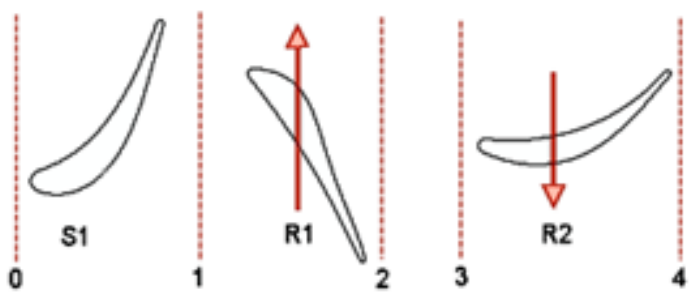

(a) $1+1 / 2$ counter-rotating turbine
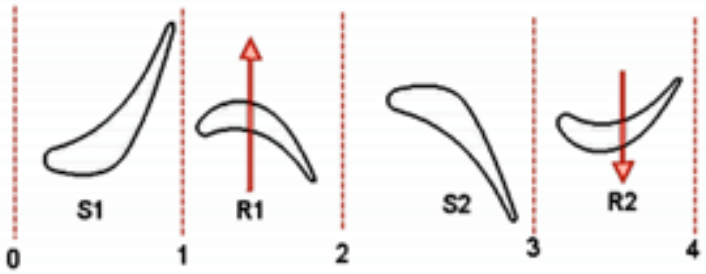

(b) $1+1$ counter-rotating turbine

Figure 1. Diagram of blade structure in a $1+1 / 2$ and $1+1$ counter-rotating turbine
The adoption of aerodynamic configurations in counter-rotating turbine is one of the most effective techniques to improve the engine performance [1]. The conter rotation of HP and LP turbine can greatly reduce or eliminate the gyroscopic moment in the engine [2] and improve the maneuverability and operability of the aircraft [3]. Moreover, it can reduce the weight of the turbine from the aspect of structure, which makes the engine structure more compact [4]. In 1985, Louis and Mossca of the MIT in the United States conducted resaearch about $1+1 / 2$ and $1 / 2+1 / 2$ counter-rotating turbine and selected the class load coefficient as the free variable, and the result showed that under the same level of load coefficient, the efficiency of the counter-rotating turbine was higher than that of the conventional turbine [5]. In 1993, Huber from NASA's lewis research center studied counter-rotating turbine in a liquid rocket engine [6] and the results showed that the efficiency of turbine without a second-level turbine guide vane increased by $2 \%$. Lior from Russia used the oblique flow combustion chamber technology in his experiment [7] to provide a certain amount of airflow prewhirl to eliminate the high pressure guide vane and finally he got a $1 / 2+1 / 2$ contrarotating turbine scheme and found that under the same flow conditions and turbine expansion ratio, the efficiency can be improved by $3 \%$ to $5 \%$ [8]. In 2000 , Haldeman [9] cooperated with $\mathrm{P} \& \mathrm{~W}$ in both an experiment and numerical simulation of a $1+1 / 2$ turbineturbine, the results showed that the low-pressure turbine surface was subjected to larger unsteady stress [10]. The 
diagram of high pressure in $1+1 / 2$ counter-rotating turbine given by Wang Huishe [11] showed that a set of compressive wave (SSPW) appeared near the $60 \%$ axial chord length on the suction surface of the high pressure rotator blade but weakened after its intersecting with the trailing edge (IES). Qi Lei from Beihang university conducted simulation about a small $1+1 / 2$ contrarotating turbine she designed and the results showed that the exit Mach number of the high pressure rotor is high, and complex waves produced in the channel with strong shock wave throughout the channel [12].

In this research, numerical simulation were carried out for a $1+1 / 2$ contra-rotating turbine under design conditions to obtain distribution regulations of the shock wave structure in blade fields, and then schlieren technique was introduced to get the detailed shock wave structure, followed by analyzation of the experimental results.

\section{Numerical simulation}

\subsection{Computational Grid}

The object of this calculation is a $1+1 / 2$ counter rotating turbine, in which the number of high pressure guide vanes is 17 , the number of high pressure rotor blades is 29 , and the number of low pressure rotor blades is 33 . Autogrid software is used to mesh the single channel model of the turbine. The grid diagram is shown in Fig. 2.

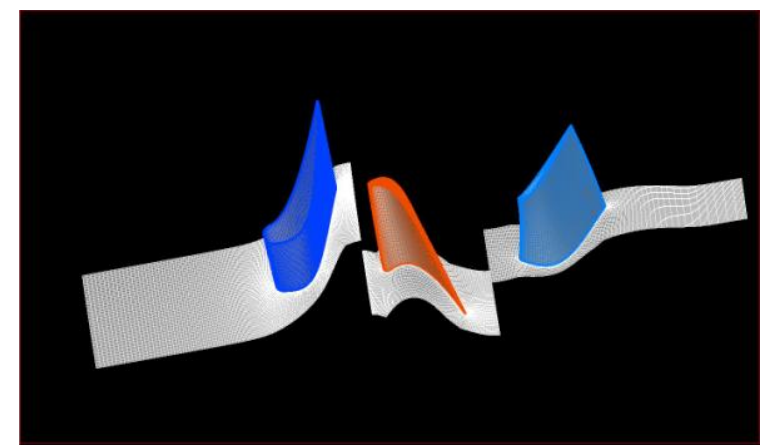

Figure 2. Grid of single channel model

The steady numerical simulation is done by software CFX15.0, and the numerical simulation method is time chasing finite volume method. The calculated refrigerant is set to simulate the gas by changing the specific heat according to the oil gas ratio. When conducting unsteady calculation, set the physical time step of turning the lowpressure rotor through 1 channel as 50 steps, and each step of the virtual time step is 10. Transient Rotor Stator is selected as the dynamic interface type.

\subsection{The Calculation Results}

Shown in Fig. 3 to 5 is the distribution of isentropic Mach number on surface of blade root, middle and tip (corresponding to $10 \%, 50 \%, 90 \%$ blade height). It can be seen from the Fig. 3 that the airflow around the surface of tip and middle of the high pressure guide vane is in subsonic flow condition, but supersonic state around the surface of posterior root. The inlet angle of attack is 0 degree and the unsteady results are in good agreement with the steady results at the the front part of the root, middle and tip part of the rotator. The suction peak near the trailing edge of suction surface of unsteady timeaverage result is located posteriorly compared with that of steady result, and the diffusivity of trailing edge increased. This is because there are various waves in this area, and the flow is complicated. It shows that the unsteady effect has a great influence on the wave flow in area of guide vane.

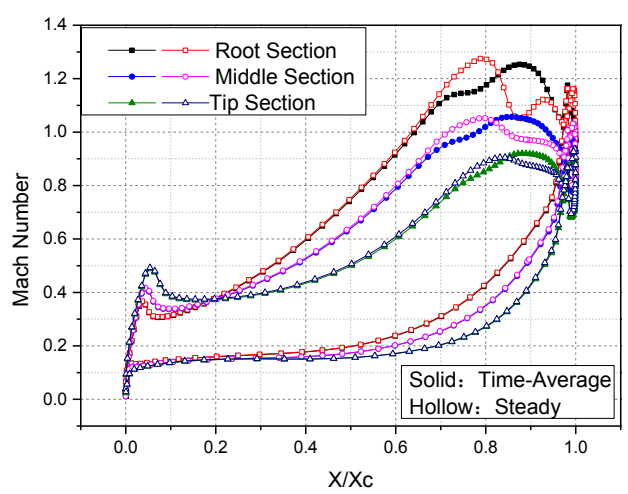

Figure 3. Distribution of Isentropic Mach number in high pressure guide vane surface under steady and unsteady conditions

In order to improve the working capacity of the low pressure rotator blade in $1+1 / 2$ contra rotating turbine, the high pressure rotator blade must have higher outlet Maher number and relative small gas flow angle. Fig. 4 shows that the high pressure rotor blade is in transonic flow state, and the section from trailing edge to the exit of blade is completely in the supersonic state. The suction peak of leading edge also produces supersonic flow due to the large degree of acceleration. It can be seen from the load state at the head of the blade that the inlet flow of the high pressure rotor has large positive angle of attack and strong shock wave structure appears because of the supersonic flow at the trailing edge of the suction side. Besides, the shock intensity decreased gradually from root to tip.

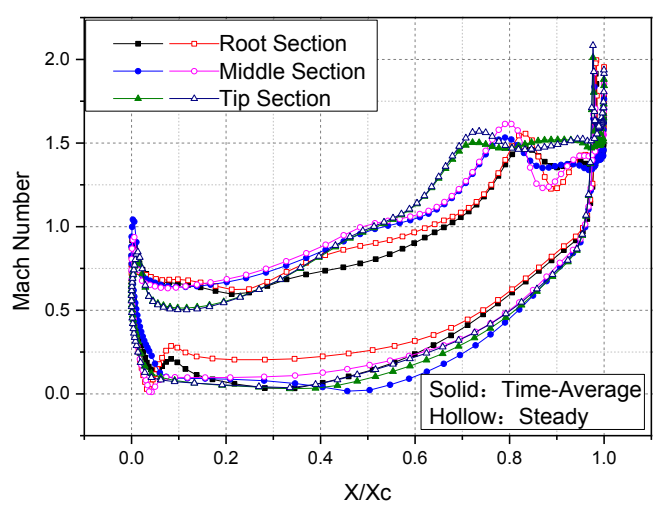

Figure 4. Distribution of Isentropic Mach number in high pressure rotor surface under steady and unsteady conditions 
Compared with the high pressure guide vane, the unsteady results are not that agreed with the steady results in high pressure rotator, which is more obvious in the front part of the blade axis. Because of the higher positive angle of attack, the unsteady isentropic Mach number is lower than that of steady result, especially at the middle pressure surface of the blade root and both the suction and pressure surface of middle section of blade.

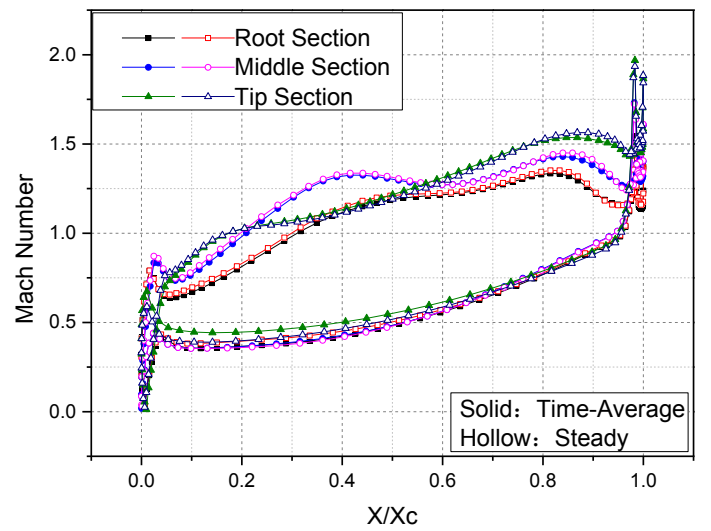

Figure 5. Distribution of Isentropic Mach number in low pressure rotor surface under steady and unsteady conditions

Fig. 5 shows that most regions in the low pressure rotor is in supersonic state, and the distribution of blade surface isentropic Mach number is relatively flat. The load distribution is more reasonable, showing better acceleration performance of airflow in the blade surface. Unlike high pressure blade, the unsteady and steady results of load distribution in low pressure rotor blade are in satisfactory agreement, except slightly upward of isentropic Mach number in the front part of the pressure side of blade tip. It can be seen that most of the areas of the low pressure rotator blade surface are less affected by the unsteady effect.

\section{The experimental study}

In order to deeply study the dynamic characteristics of the shock wave structure of the designed high pressure guide vane, high pressure and low pressure blade under the supersonic flow condition, $50 \%$ height section of the blade of the three types of blades are extracted respectively to make plane cascades shown in Fig. 6, which are conducted blowing experiments in transonic wind tunnel by using the schlieren display technology to record the shock wave structure in three cascade flow passage under the design Angle of attack.

The ratio of inlet total pressure and outlet static pressure was used as the experimental condition variable, and according from the designed outlet Mach number of the turbine, the blow down pressure ratio was set to change from 1.9 to 2.5 , and the experimental results were compared with the results of cascade steady numerical calculation.

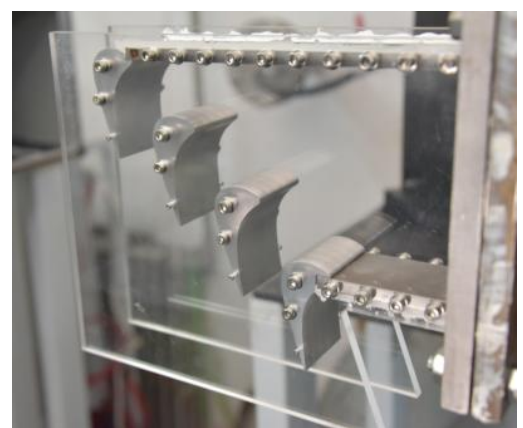

Figure 6. Plane cascade

\subsection{Experimental Equipment}

This experiment was conducted on supersonic wind tunnel of Beihang University, schlieren technology was adopted to observe the wave structure in the blade cascade, and the atmospheric pressure and the total inflow pressure were recorded at the experimental steady state. The experiment system is shown as belowing Fig. 7.

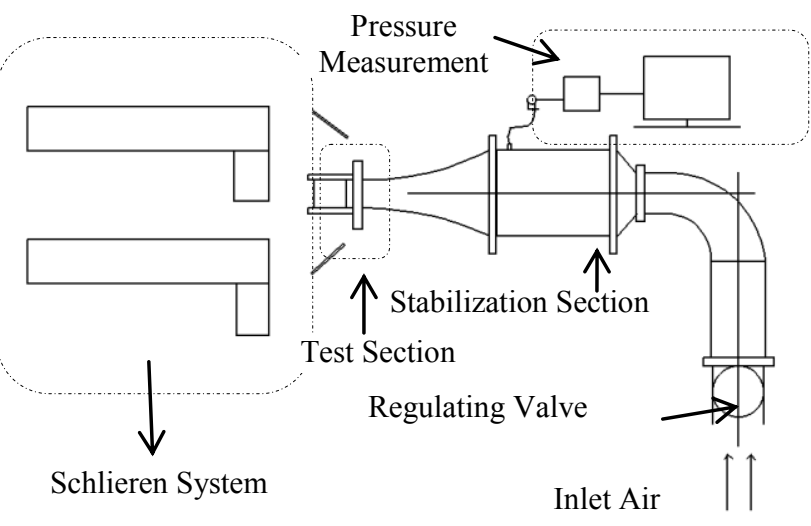

Figure 7. Experiment system

The experimental device consists of seven parts: the air source, pneumatic valve, expansion section, stabilization section, convergence section, test section and gas collecting silencer. The airflow contractes into a rectangular $120 \mathrm{~mm} \times 50 \mathrm{~mm}$ channel after the convergent section, then it flows through the test section, and finally into the atmosphere through the gas collecting silencer.

For the schlieren device of this experiment shown above in Fig. 8, a double reflection schlieren instrument, which is a typical parallel light schlieren instrument, was adopted.

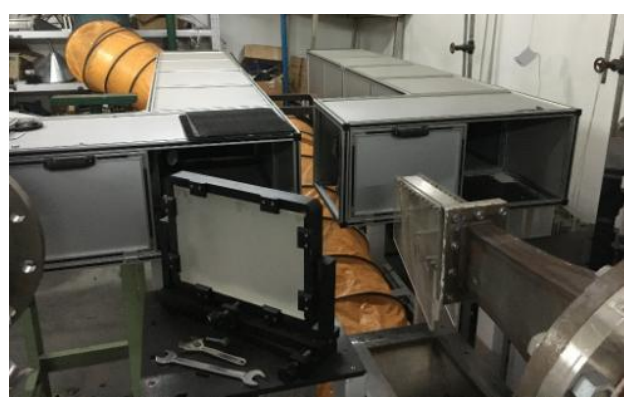

Figure 8. Double reflection schlieren instrument 


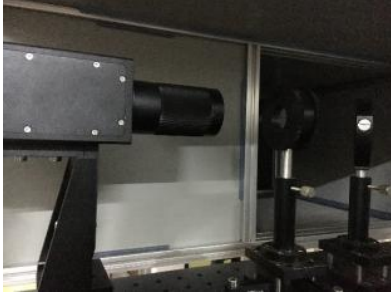

(a) Light source system

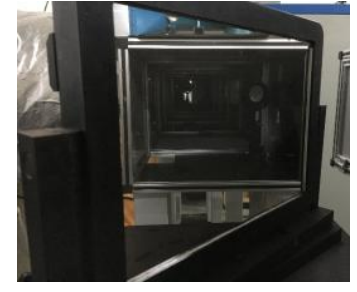

(b) Main light path

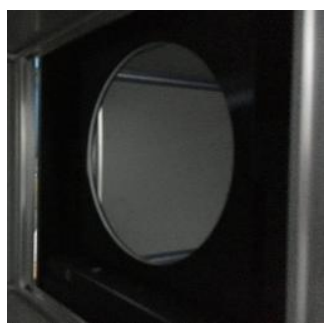

(c) Concave mirror

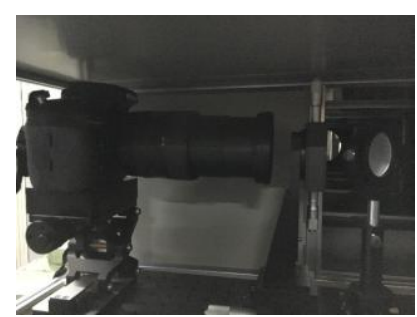

(d) Captured system
Figure 9. Components of schlieren system

The components are shown in the following Fig. 9. The concave reflection mirrior has a diameter of $200 \mathrm{~mm}$, a focal length of $2000 \mathrm{~mm}$, and the image is taken with a Nikon D810 SLR camera. Camera shutter time is set to $1 / 1000$, and the aperture is F5.6, ISO200.

\subsection{Experimental Results}

Given below are the numerical simulation results and schlieren test results of the three sets of high pressure guide vanes, high pressure rotator blades and low pressure rotator blades under the blow down ratio of 1.9 , $2.1,2.3$ and 2.5 respectively.

It can be seen from the diagram that the related wave structure is obvious, and the internal tail wave, the outer tail wave and the reflected wave of the inner tail wave can be clearly seen. The suction surface of the upper channel has no flow, which has a great influence on the flow inside the channel, so that the two channels of the upper and lower channels lose periodicity. Therefore, the analysis of flow characteristics will mainly focus on the intermediate runner.

Given belowing are simulation and experimental results of high pressure rotor. From the schlieren results in Fig. 10 we can see that with the increase of the pressure ratio, the Mach number of the three sets of the cascade increases gradually, and the structure of the shock wave tends to be more complex. When the Mach number is 1.9 ,where is no obvious shock at exit of the cascade and when the pressure ratio reaches 2.1 , we can see obvious inner tail wave structure and its reflection wave on the adjacent suction surface, while intensity of the outer tail wave is relatively weak.

As the pressure ratio increases, the shock wave structure at the exit of cascade gradually increases, and you can see the position of the inner tail wave hitting on the adjacent blade gradually moves toward the direction of exports, while the reflection wave of tail wave gradually increases. However, the trend of changes of the wave structure is overall weak.

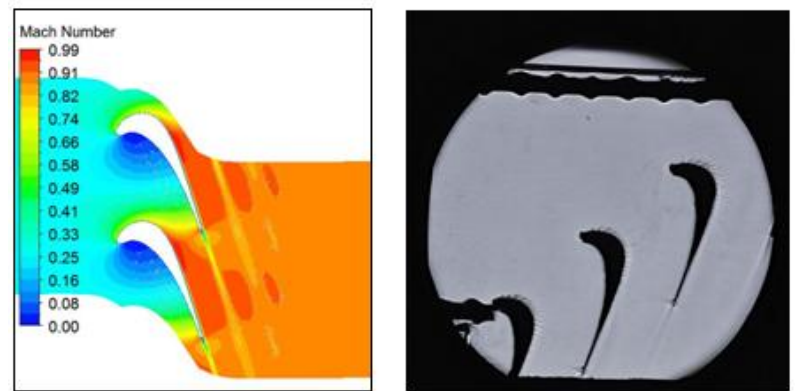

(a) $\mathrm{P} * / \mathrm{P}_{\mathrm{b}}=1.9$

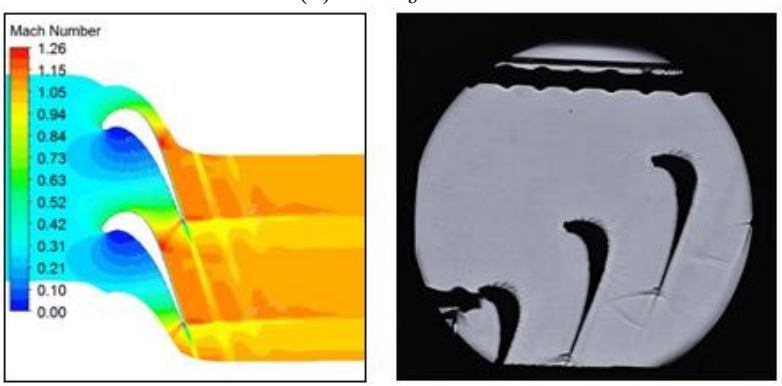

(b) $\mathrm{P} * / \mathrm{P}_{\mathrm{b}}=2.1$

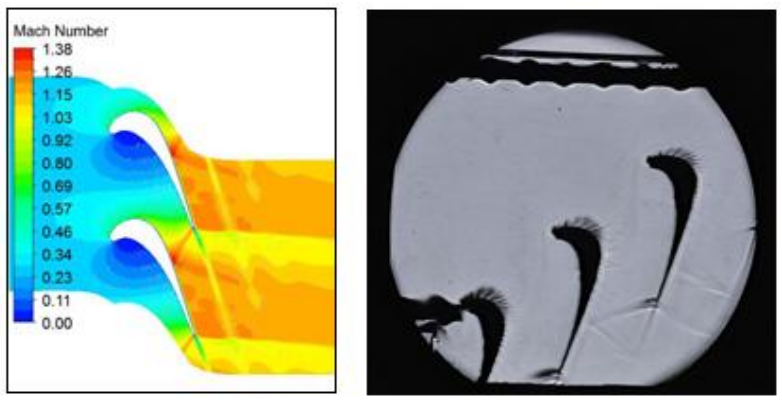

(c) $\mathrm{P} * / \mathrm{P}_{\mathrm{b}}=2.3$

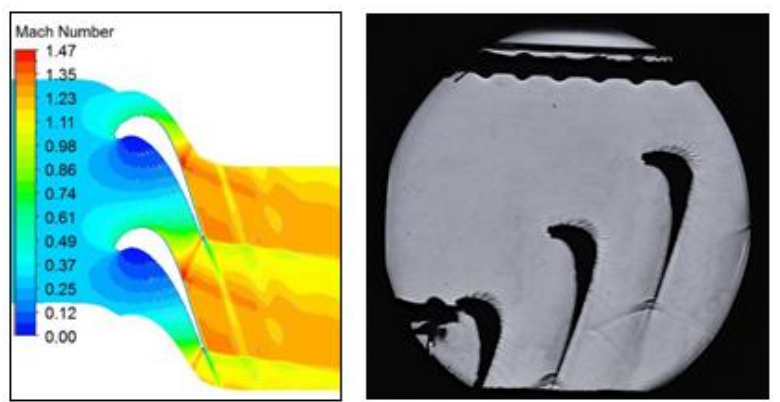

(d) $\mathrm{P} * / \mathrm{P}_{\mathrm{b}}=2.5$

Figure 10. Simulation and Experimental results of high pressure rotor

The numerical simulation results of low pressure rotating blades under different pressure ratios and the corresponding schlieren shock wave structure are shown below in Fig. 11. It can be seen clearly that with the increasing of the pressure ratio, the shock wave structure of the low pressure blade is gradually increasing. When the pressure ratio is 1.9 , we can see that the trailing edge shock wave is weak and only an inner tail wave and an external tail wave can be seen without reflection waves. When the pressure ratio is 2.1 , the reflection wave of the inner tail wave can be clearly seen, but the intensity is weak. When the pressure ratio reaches 2.3 as shown below, we can see the reflection wave of outer tail wave 
$\mathrm{B}$ and the reflection wave of inner tail wave A on the adjacent blade clearly. The intensity of internal reflection wave is not obviously weakened after intervening with tail wake $\mathrm{F}$, and second reflection wave is produced after hitting the upstream wake again. The intensity of the outer tail wave of the downstream blade is weakened obviously after the interference with the wake F.
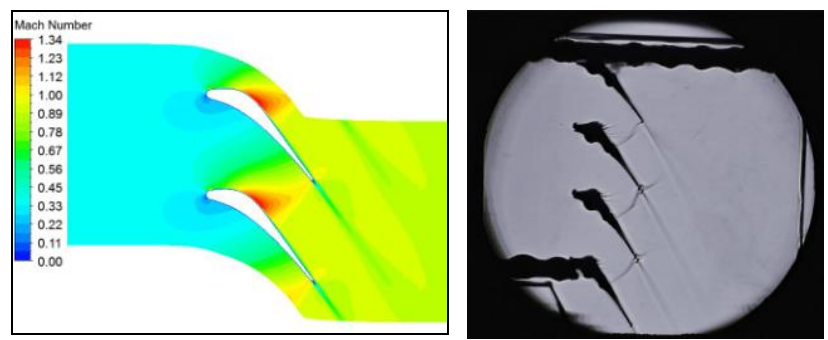

(a) $\mathrm{P} * / \mathrm{P}_{\mathrm{b}}=1.9$
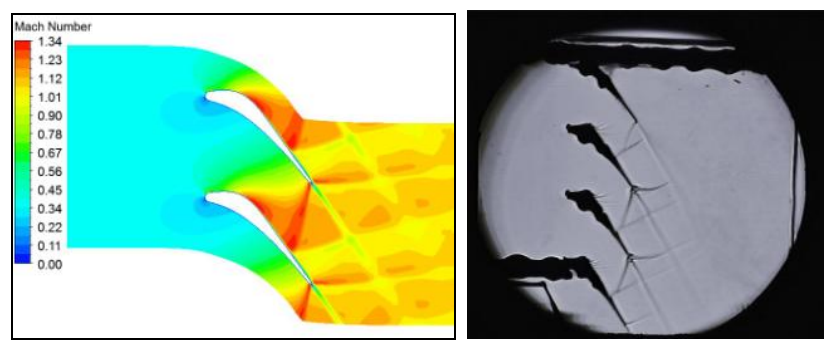

(b) $\mathrm{P} * / \mathrm{P}_{\mathrm{b}}=2.1$
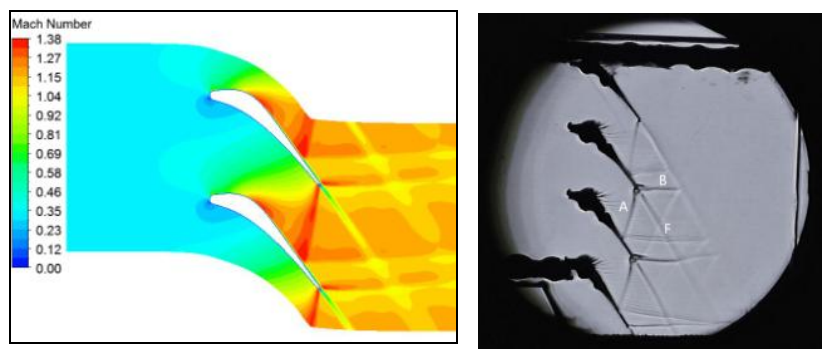

(c) $\mathrm{P} * / \mathrm{P}_{\mathrm{b}}=2.3$
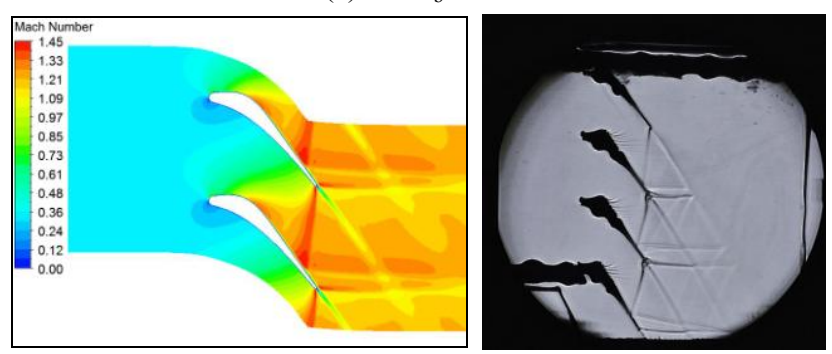

(d) $\mathrm{P} * / \mathrm{P}_{\mathrm{b}}=2.5$

Figure 11. Simulation and Experimental results of low pressure rotor

When the pressure ratio is 2.5 , we can clearly see the wave structure moving toward the exit direction, and the wave structure is more complex. The strength of the tail waves and their reflection waves significantly enhances, and multiple reflection waves are produced after the interference between the wake and the primary reflection waves, which brings a great energy loss. The relative position change and intensity enhancement of the outer tail wave and the inner tail wave can be observed obviously by the contrast Fig. 12 of the pressure ratio 2.3 and 2.5 .

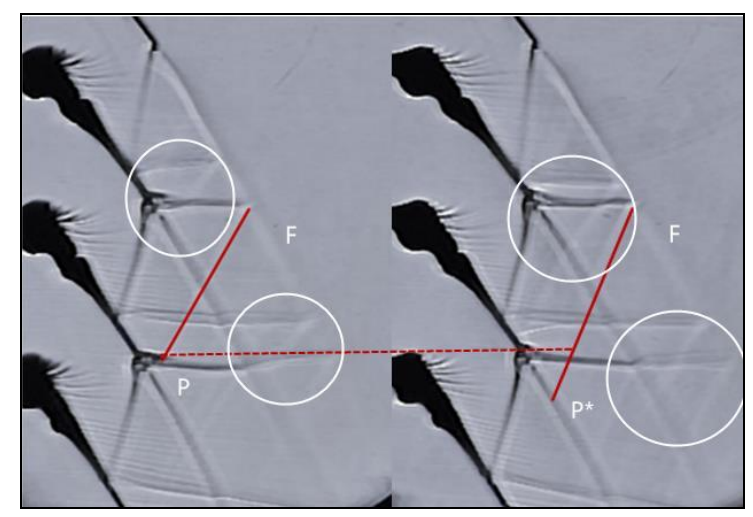

Figure 12. A comparison of wave structures details of LP rotor under pressure ratio 2.3 and 2.5

In the two figures, the positions of intersection points of the reflected wave of the outer tail wave and the wakes are made respectively, and horizontal lines are made to compare the differences of horizontal position between the two points. You can clearly see the intersection points moving toward the exit direction when the pressure ratio increases to 2.5, and the strength of both outer and inner tail wave significantly improves with increase of pressure ratio. At the same time in the white circle position the enhancement of intensity of the reflection wave also can be seen clearly, and when the pressure ratio is 2.5 , the stronger reflection wave of the upstream blade mixes together with the reflection wave of the inner tail wave to form a new stronger reflection wave. At this moment, the wave structure is complicated which brings larger shock loss.

Shown in following Fig. 13 are distributions of the shock wave structure in high pressure guide vanes at different pressure ratios. As the blade thickness is small, the stress change caused by laser drilling has a great influence on the density change of the glass plate around the hole. But the wave structure is quite obvious, and primary shock wave from the suction surface and its reflected wave on the adjacent blade can be seen at the pressure ratio of 2.1. Besides, the reflection wave of inner tail wave hitting on the adjacent blade surface reflects again after the interference with the wake. All those waves and reflection waves, as well as the expansion wave system of the trailing edge of the pressure surface, form a complex wave structure system.
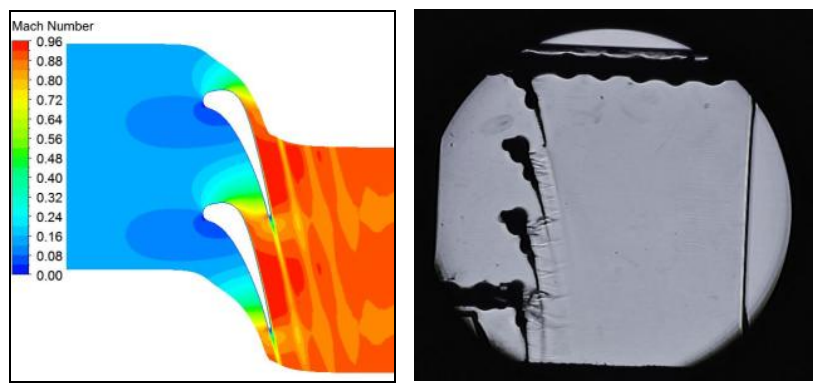

(a) $\mathrm{P} * / \mathrm{P}_{\mathrm{b}}=1.9$ 

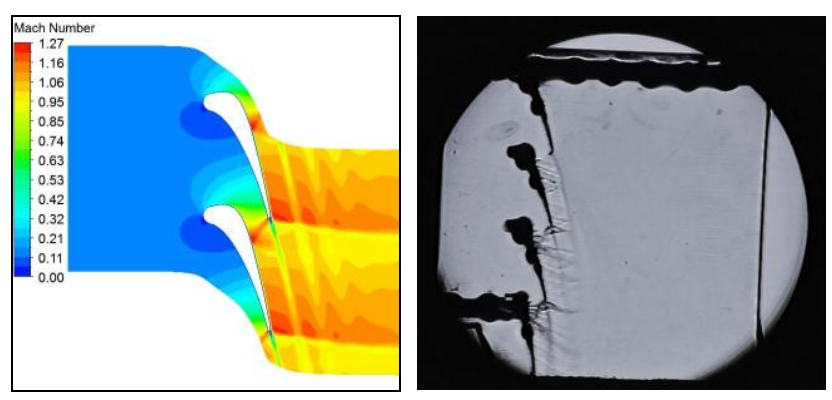

(b) $\mathrm{P} * / \mathrm{P}_{\mathrm{b}}=2.1$
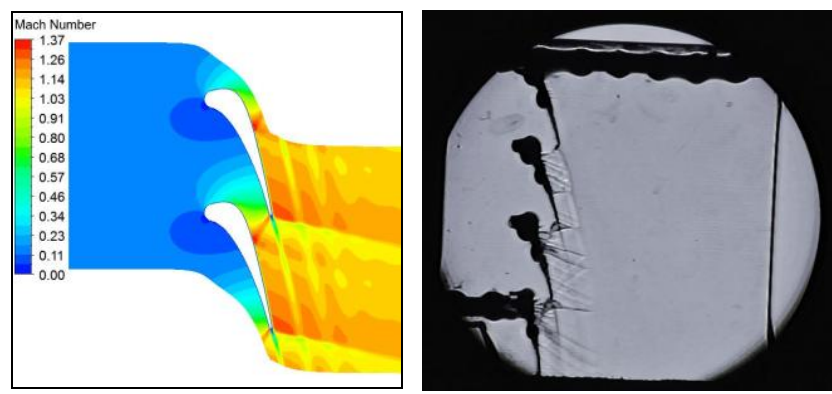

(c) $\mathrm{P} * / \mathrm{P}_{\mathrm{b}}=2.3$
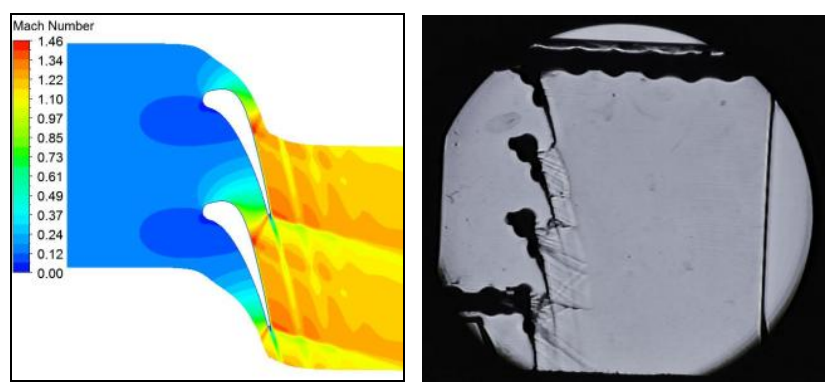

(d) $\mathrm{P} * / \mathrm{P}_{\mathrm{b}}=2.5$

Figure 13. Simulation and Experimental results of high pressure guide vane

\section{Conclusion}

(1) The unsteady effect has little influence on the flow filed of high pressure guide vane, while the wake of high pressure guide vane periodically sweeps the leading edge of high pressure rotating blade, which brings strong unsteady characteristics and periodic variations of exit Mach number and exit shock wave.

(2) The distribution of pressure in high pressure rotor flow field shows periodic variation along with time, and strong unsteady aerodynamic force appears on the surface of low pressure rotor blade under the periodic effect of not only strong shock wave outside the rotor trailing edge but also strong wake and potential effects. The fluctuations of pressure in the channel of low pressure rotor is extremely volatile.

(3) As the pressure ratio increases, the exit Mach number increases and the wave structure in blade channel gradually moves to the outlet direction. The interference between the outer tail wave and adjacent blade wake is gradually strengthened as well as the reflection wave of internal tail wave, but the overall changing trend of wave structure in rotor channel is weak. On the contrary, the wave structure system in high pressure guide vane changes more severe as the pressure ratio rises, and the primary shock wave and expansion wave significantly enhanced with the increasing of pressure ratio.

\section{References}

1. Macioce L.E., Schaefer J.W., Saunders N.T. A Status Report on the Energy Efficient Engine Project[R], NASA-TM-81566, (1980).

2. Koop W. The Integrated High Performance Turbine Engine Technology (IHPTET) Program[A]. ISABE 97-7175, (1997)

3. Kauser, Fazal B.. An Overview of Gas Turbine Propulsion Technology[A]. AIAA Paper[C], AIAA1994-2828. (1994)

4. Maclin H., Krause F.. Propulsion Technology for Future Commercial Aircraft[A]. AIAA Paper[C], AIAA-2003-2544. (2003)

5. Lious J.F.. Axial Flow Contra-Rotating Turbines[A]. ASME Paper[C], 85-GT-218, (1985)

6. Hubber F.W., Branstrom B.R., Finke A.K., et al. Design and Test of a Small Two Stage CounterRotating Turbine for Rocket Engine Application[A]. AIAA Paper[C], AIAA-93-2136, (1993)

7. Lior D.,Priampolsky R. Design of Stator-Less Turbine[R]: Becker System Engineering PPT (2004)

8. Shafer D.G., McNelis N.. Development of a Group Based Mach 4+ Revolutionary Turbine Accelerator Technology Demonstrator (RTATD) for Access to Space[A]. ISOABE[C],( 2003)

9. Haldeman C.W., Dunn M.G., Abhari R.S., et al. Experimental and Computational Investigation of the Time-Resolved Pressure Loading on a Vaneless Counter-Rotating Turbine[A]. ASME Paper[C], 2000-GT-0445. (2000)

10. Weaver M.M., Manwaring S.R., Abhari R.S., et al. Forcing Function Measurements and Preditions of a Transonic Vaneless Counter-rotating Turbines[A]. ASME Paper[C], 2000-GT-375 (2000)

11. H S Wang, H Zhang, Q J Zhao, et al. numerical study of the flow field at the top clearance of rotating rotor in a $1+1 / 2$ turbine $[\mathrm{J}]$. Journal of Engineering Physics, 28 (5): 751-754 (2007)

12. L Qi, S N Pan, Livy et al. unsteady flow numerical simulation of $1+1 / 2$ Contra-Rotating turbine [J]. Journal of aerospace power, 25 (8): 1825-1835. (2010) 